\title{
Aliskiren targets multiple systems to alleviate cancer cachexia
}

\author{
CHAOYI WANG ${ }^{1 *}$, DUNWEI GUO ${ }^{1 *}$, QIANG WANG ${ }^{1,2^{*}}$, SONG YOU ${ }^{1}$, \\ ZHONGPENG QIAO $^{1}$, YONG LIU ${ }^{1}$, HANG DAI ${ }^{1}$ and HUA TANG $^{1}$ \\ ${ }^{1}$ Department of Gastrointestinal Surgery, The First Affiliated Hospital of Chongqing Medical University, Chongqing 400016; \\ ${ }^{2}$ Department of Gastrointestinal Surgery, Suining Central Hospital, Suining, Sichuan 629000, P.R. China
}

Received February 18, 2016; Accepted March 26, 2016

DOI: $10.3892 /$ or.2016.5118

\begin{abstract}
To examine the effects of aliskiren, a small-molecule renin inhibitor, on cancer cachexia and to explore the underlying mechanisms. A cancer cachexia model was established by subcutaneously injecting $\mathrm{C} 26$ mouse colon carcinoma cells into isogenic BALB/c mice. Aliskiren was administered intragastrically $[10 \mathrm{mg} / \mathrm{kg}$ body weight $(\mathrm{BW})]$ on day 5 (as a preventive strategy, AP group) or on day 12 (as a therapeutic strategy, AT group) after C26 injection. Mice that received no C26 injection (healthy controls, HC group) or only C26 injection but not aliskiren (cancer, CA group) were used as controls. $\mathrm{BW}$, tumor growth, whole body functions, and survival were monitored daily in half of the mice in each group, whereas serum, tumors, and gastrocnemius muscles were harvested from the other mice after sacrifice on day 20 for further analysis. Aliskiren significantly alleviated multiple cachexia-associated symptoms, including BW loss, tumor burden, muscle wasting, muscular dysfunction, and shortened survival. On the molecular level, aliskiren antagonized cachexia-induced activation of the renin-angiotensin system (RAS), systematic and muscular inflammation, oxidative stress, and autophagy-lysosome as well as ubiquitin-proteasome stimulation. In addition, early administration of aliskiren before cachexia development (AP group) resulted in more robust effects in alleviating cachexia or targeting underlying mechanisms than administration after cachexia development (AT group). Aliskiren exhibited potent anti-cachexia activities. These activities were achieved
\end{abstract}

Correspondence to: Dr Hua Tang, Department of Gastrointestinal Surgery, The First Affiliated Hospital of Chongqing Medical University, Chongqing 400016, P.R. China

E-mail: tanglihua6969@sina.com

"Contributed equally

Abbreviations: RAS, renin-angiotensin system; ALP, autophagy-lysosome pathway; UPP, ubiquitin-proteasome pathway; $\mathrm{H} \& \mathrm{E}$, hematoxylin and eosin; SOD, superoxide dismutase; GSH-Px, glutathione peroxidase; MDA, malondialdehyde; RIPA, radioimmunoprecipitation assay; PMSF, phenylmethylsulfonyl fluoride; SDS, sodium dodecyl sulfate; PAGE, polyacrylamide gel electrophoresis; PVDF, polyvinylidene fluoride

Key words: cancer cachexia, aliskiren, renin-angiotensin system, inflammation, oxidative stress through the targeting of at least four mechanisms underlying cachexia development: RAS activation, increase in systematic inflammation, upregulation of oxidative stress, and stimulation of autophagy-lysosome pathway (ALP) and ubiquitin-proteasome pathway (UPP).

\section{Introduction}

Cancer cachexia is a complicated multifactorial syndrome featuring a progressive loss of skeletal muscle mass (with or without fat loss) that is associated with significant functional impairments (1). Cachexia occurs in $\sim 60 \%$ of patients with advanced cancer and up to $80 \%$ of those in a terminal stage $(2,3)$. The development of cachexia is divided into three stages: pre-cachexia, cachexia, and refractory cachexia. Cachexia significantly and adversely impacts the patient responses to treatment, quality of life, and survival. Cachexia is responsible for $20-30 \%$ of all cancer-related deaths (2). No treatments effectively alleviating cachexia have been developed to date, and thus, it is essential to explore its pathogenesis and underlying mechanisms, and develop therapeutic approaches targeting these mechanisms.

Muscle wasting is a prominent feature of cachexia and is closely associated with increased protein degradation. Studies have identified several mechanisms modulating protein degradation in muscles. One is the renin-angiotensin system (RAS): although most commonly associated with vasoconstriction and the regulation of blood pressure, angiotensin peptides angiotensin I (Ang I) and II are produced within skeletal muscle and directly or indirectly inhibit protein synthesis (4), promote protein degradation (5), stimulate cytokine production $(6,7)$, and generate hydroxyl radicals $(\bullet \mathrm{OH})$ to exacerbate oxidative stress $(8,9)$. Consistent with its functional consequences, the RAS is noted to be activated in conditions involving muscle wasting, including sarcopenia, chronic heart failure, chronic renal failure, and cancer (10-13). Our previous study as well as that from Murphy et al (14), demonstrated the significance of the RAS in cancer cachexia (15), corroborating the potential of a RAS inhibitor/antagonist in treating cancer cachexia. A second mechanism is the autophagy-lysosome pathway (ALP) and ubiquitin-proteasome pathway (UPP): both are major pathways responsible for muscular protein degradation $(16,17)$. Examples of genes critical for regulating these two machineries in muscles include Bnip3, Beclin1, and LC3, which are involved in different steps of autophagosome formation, 
and two muscle-specific ubiquitin E3 ligases, Atrogin1 and MuRF1 (17).

In this study, we examined the efficacy of a novel direct renin inhibitor, aliskiren, on cancer cachexia. Previous studies showed that aliskiren can potently inhibit inflammation, reduce oxidative stress, and protect against tissue damage (18-20). However, minimal information is available on its actions in cancer cachexia. To address this issue, we established a cancer cachexia mouse model, assessed the prophylactic as well as therapeutic effects of aliskiren on cancer cachexia, and explored the molecular mechanisms underlying these effects.

\section{Materials and methods}

Ethics statement. The experimental protocols involving human samples were established according to the ethical guidelines of the Helsinki Declaration and approved by the Ethics Committee of Chongqing Medical University (Chongqing, China). The experimental protocols involving mice were approved by the Institutional Animal Care and Use Committee (IACUC) of Chongqing Medical University.

Cells and reagents. C26 mouse colon carcinoma cells were provided by the Research Center (the First Affiliated Hospital of Chongqing Medical University, Chongqing, China) and cultured in Dulbecco's Modified Eagle Medium (DMEM)-F12 supplemented with $10 \%$ fetal bovine serum (FBS; both from Hyclone, Logan, UT, USA).

Aliskiren was purchased from Novartis (San Diego, CA, USA). The following antibodies were used in this study: anti-Bnip1 (ab38621), anti-LC3 (ab168831; Abcam, Cambridge, MA, USA), anti-MuRF1 (sc-32920), anti-Atrogin1 (sc-33782; Santa Cruz Biotechnology, Santa Cruz, CA, USA), anti-Beclin1 (11306-1-AP), anti-GAPDH (10494-1-AP), and peroxidase-conjugated anti-rabbit IgG (SA00001-2; Proteintech, Wuhan, China). A real-time polymerase chain reaction $(\mathrm{PCR}) \mathrm{kit}$ was purchased from Takara (Dalian, China), and a western immunoblot kit was purchased from Beyotime (Jiangsu, China).

Experimental animals and cachexia model. Healthy male BALB/c mice between 7 and 9 weeks of age and with a body weight (BW) of 21-26 g were purchased from the Experimental Animal Center (Chongqing Medical University). All mice were housed in a specific-pathogen-free facility at room temperature of $22 \pm 1^{\circ} \mathrm{C}$ on a $12 / 12$-h light/dark cycle with access to food and water ad libitum.

To induce cancer cachexia, C26 cells growing in log phase were detached and resuspended as single cells in PBS at $1 \times 10^{7} / \mathrm{ml}$, and $100 \mu \mathrm{l} \mathrm{C26}$ cell suspension was injected subcutaneously into the left axilla of each mouse $(\mathrm{N}=48)$. In healthy controls (HC group), $100 \mu \mathrm{l}$ PBS was injected into the mice $(n=16)$. Mice receiving C26 injection were further divided into three groups ( $n=16 /$ group): i) cancer-only group (CA): mice received PBS intragastrically on days 5 and 12 after C26 injection; ii) aliskiren prevention group (AP): mice received aliskiren $(10 \mathrm{mg} / \mathrm{kg} \mathrm{BW})$ in PBS intragastrically on day 5 after C26 injection, when tumor nodules were palpable; and iii) aliskiren treatment group (AT): mice received aliskiren (10 mg/kg BW) in PBS intragastrically on day 12 after C26 injection, when cachexia was well-developed. Following C26 cell injection, all mice were monitored daily for the appearance of skin and hair, mental state, BW, and tumor growth until day 20, when eight mice from each group were sacrificed by $\mathrm{CO}_{2}$ euthanasia followed by cervical dislocation for sample collection. The tumor volume was calculated as $\mathrm{V}=\mathrm{ab}^{2} / 2$, where $\mathrm{V}$ is the tumor volume and $\mathrm{a}$ and $\mathrm{b}$ are the length and width of the tumor, respectively. The other eight from each group were continued to be monitored daily and used to assess whole-body functions (as described below) and survival time. According to the IACUC guidelines of Chongqing Medical University, mice were euthanized by $\mathrm{CO}_{2}$ asphyxiation followed by cervical dislocation when they presented signs of moribund conditions, including decreased response to stimuli, inability to remain upright, abnormal feeding behavior, evidence of severe muscle atrophy, and rough hair coat.

Assessment of grip strength, coordination, and locomotor activity. Whole body strength and coordination was assessed on day 20 after C26 cell injection using a TSE Grip Strength Meter and the rotarod performance test RotaRod Advanced (both from TSE Systems, Germany) as previously described (21-23). Briefly, to measure grip strength, the mouse was allowed to hold on firmly to a grip that was mounted to a high-precision force sensor, and its tail was pulled backwards with a continuous movement until the grip was broken. The force value at this point was recorded. Ten measurements were taken for each animal within 2 min, and the maximum values were used for statistical analysis. For rotarod performance test, the mouse was placed on a horizontal rod that rotated from a starting speed of $4 \mathrm{rpm}$ and accelerated at $1 \mathrm{rpm} / 8 \mathrm{sec}$ until the mouse fell off the rod. The latency-to-fall was recorded. The test was repeated three times within 15 min for each animal, and the longest latency-to-fall was used for statistical analysis.

Locomotor activity was monitored using an infrared monitoring system (WV-CF314LCH; Panasonic, Japan) following the manufacturer's instructions.

Sample collection. On day 20 after C26 inoculation, eight mice from each group were sacrificed, with blood immediately collected from the retro-orbital venous sinus. The blood samples were allowed to sit at $4^{\circ} \mathrm{C}$ for $1 \mathrm{~h}$, which was followed by centrifugation at $1,006 \mathrm{x}$ for $10 \mathrm{~min}$ at $4^{\circ} \mathrm{C}$. The serum supernatant was then collected and stored at $-80^{\circ} \mathrm{C}$ until future use. In addition to the serum sample, the tumor tissue as well as the bilateral gastrocnemius muscles were isolated and weighed. The left gastrocnemius muscle was fixed in $4 \%$ paraformaldehyde, and the right one was flash frozen and stored in liquid nitrogen until further use.

Measurement of cross-sectional area of gastrocnemius muscle. After $48 \mathrm{~h}$ of fixation in paraformaldehyde, the gastrocnemius muscle was embedded in paraffin, sectioned onto glass slides, and stained with hematoxylin and eosin (H\&E) (Beyotime). Three slides for each sample were imaged under an optical microscope (x200 magnification, BX51TF; Olympus, Tokyo, Japan), with five random fields imaged for each slide, and the cross-cut area of the gastrocnemius muscle was measured using Image-Pro Plus 6.0 software (Media Cybernetics, Rockville, MD, USA). 
Table I. Primer sequences used in real-time polymerase chain reaction (PCR) analysis.

\begin{tabular}{lll}
\hline Gene & \multicolumn{1}{c}{ Sense primer $\left(5^{\prime} \rightarrow 3^{\prime}\right)$} & \multicolumn{1}{c}{ Antisense primer $\left(5^{\prime} \rightarrow 3^{\prime}\right)$} \\
\hline Bnip3 & 5'-GGGTTTTCCCCAAAGGAATA-3' & 5'-TGACCACCCAAGGTAATGGT-3' \\
LC3 & 5'-CGGCTTCCTGTACATGGTTT-3' & 5'-ATGT GGGTGCCTACGTTCTC-3' \\
Beclin1 & 5'-ATGGAGGGGTCTAAGGCGTC-3' & 5'-TGGGCTGTGGTAAGTAATGGA-3' \\
MuRF1 & 5'-GGAACACGAAGACGAGAAAATC-3' & 5'-TGGCTATTCTCCTTGGTCACTC-3' \\
Atrogin1 & 5'-GAAGAGAGCAGTATGGGGTCAC-3' & 5'-CTTGAGGGGAAAGRGAGACG-3' \\
Caspase-3 & 5'-TGGGACTGATGAGGAGATGGC-3' & 5'-TGCTGCAAAGGGACTGGATG-3' \\
GAPDH & 5'-GGTGAAGGTCGGTGTGAACG-3' & 5'-CTCGCTCCTGGAAGATGGTG-3' \\
\hline
\end{tabular}

Determination of serum Ang I and II levels and both serum and muscular tumor necrosis factor- $\alpha(T N F-\alpha)$ and interleukin-6 (IL-6) levels. To prepare muscle lysates, flash frozen muscles were suspended in buffer containing $10 \mathrm{mM}$ HEPES (pH 7.9), $10 \mathrm{mM} \mathrm{KCl,} 2 \mathrm{mM} \mathrm{MgCl}, 0.1 \mathrm{mM}$ EDTA (pH 8.0), $1.0 \mathrm{mM}$ DTT, $10 \% \mathrm{NP}-40$, and $0.5 \mathrm{mM}$ phenylmethylsulfonyl fluoride (PMSF), and immediately homogenized on ice. The homogenized muscle tissue was centrifuged at 16,099 $\mathrm{x}$ g for $10 \mathrm{~min}$ at $4^{\circ} \mathrm{C}$, and the supernatant was collected for future use. The levels of Ang I and II in serum and those of TNF- $\alpha$ and IL-6 in serum and in gastrocnemius muscle were determined using enzyme-linked immunosorbent assay (ELISA) kits (R\&D Systems, Minneapolis, MN, USA) following the manufacturer's instructions.

Evaluation of oxidative stress in gastrocnemius muscle. To assess the oxidative stress level in gastrocnemius muscles, the activities of superoxide dismutase (SOD) and glutathione peroxidase (GSH-Px) and the levels of $\cdot \mathrm{OH}$ and malondialdehyde (MDA) were determined using the corresponding test kits (Nanjing Jiancheng Biological Engineering Institute, Nanjing, China) following the manufacturer's instructions.

Real-time PCR analysis. Total RNA was extracted from flash frozen gastrocnemius muscles using TRIzol reagent (Takara) according to the manufacturer's instructions and reversed transcribed into cDNA. The real-time PCR reaction was set up using SYBR Premix Ex Taq II kit (Takara) and performed on an ABI Prism 7500 Sequence Detection System (Applied Biosystems, Foster City, CA, USA). The primer sequences used for real-time PCR analysis are listed in Table I. GAPDH was used as the internal control. The quantification of real-time PCR data was performed using the $2^{-\Delta \Delta C t}$ method, as previously described (24).

Western blotting. Total proteins from the flash frozen gastrocnemius muscle samples were extracted using radioimmunoprecipitation assay (RIPA)/PMSF lysis buffer (Beyotime). Equal amounts of total protein from different samples were separated by $10 \%$ sodium dodecyl sulfate (SDS)-polyacrylamide gel electrophoresis (PAGE), transferred to polyvinylidene fluoride (PVDF) membrane (Beyotime), probed with primary antibodies at $4{ }^{\circ} \mathrm{C}$ overnight, followed by incubation with corresponding secondary antibodies. Signal detection and quantification were achieved using the UVP Bioimaging System (UVP, Inc., Upland, CA, USA). The relative protein level was expressed as the ratio of signal density of a target protein to that of the internal control protein (GAPDH).

Statistical analysis. Statistical analysis was performed using SPSS 17.0 software (SPSS, Inc., Chicago, IL, USA). Quantitative data were expressed as means \pm standard deviation (SD). Differences between two groups were analyzed using Student-Newman-Keuls q-test, and those among multiple groups were analyzed using one-way analysis of variance (ANOVA). Survival analysis was performed using the log-rank test. $\mathrm{P}<0.05$ was considered statistically significant.

\section{Results}

Aliskiren delays cachexia development, reduces tumor burden, and prolongs mouse survival. To examine the effects of aliskiren on cancer cachexia and the underlying mechanisms, we subcutaneously injected C26 colon cancer cells into isogenic $\mathrm{BALB} / \mathrm{c}$ mice, a well-established experimental model of cancer cachexia (25). On day 5 after C26 cell inoculation, although tumor nodules were palpable through the skin, no significant differences in BW were noticed among the four groups of mice. On day 12, mice in the CA and AT groups showed obvious signs of cachexia, including rough skin, disheveled fur, reduced motility, and dramatic weight loss $(\mathrm{P}<0.05$, compared with mice in the $\mathrm{HC}$ group). By day 20, mice in the CA group presented the most dramatic weight loss $(\mathrm{P}<0.01$, compared with mice in the HC group), followed by those in the AT group $(\mathrm{P}<0.01$, compared with mice in the HC group; $\mathrm{P}<0.05$, compared with mice in the CA group), and the least weight loss was observed in mice of the AP group $(\mathrm{P}<0.01$, compared with mice in the $\mathrm{CA}$ group; $\mathrm{P}<0.05$, compared with mice in the AT group; Fig. 1A). Consistently, the percentage decrease in tumor-free body mass from day 0 to 20 was most significant in mice in the CA group (32.67\%), and this percentage was significantly higher than that in the AT group (21.21\%; $\mathrm{P}<0.05$, compared with the CA group) or the AP group (12.42\%; $\mathrm{P}<0.01$, compared with the $\mathrm{CA}$ group; $\mathrm{P}<0.05$, compared with the AT group; Fig. 1B).

With respect to tumor growth, aliskiren as a treatment agent (as in the AT group) or an early prevention strategy (as in the AP group) led to reductions in both tumor mass and tumor volume $(\mathrm{P}<0.05$, compared with those in the CA group), with the most robust antitumor effects observed in the AP group $(\mathrm{P}<0.01$, compared with mice in the $\mathrm{CA}$ group; $\mathrm{P}<0.05$, compared with mice in the AT group; Fig. 1C-E). 
A
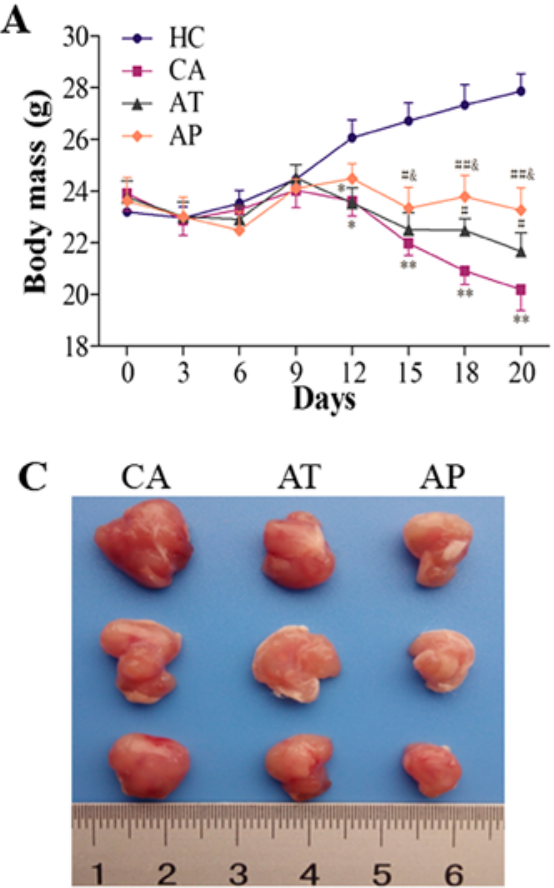

$\mathbf{E}$

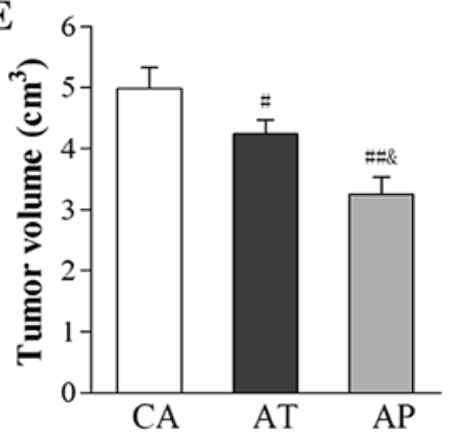

B

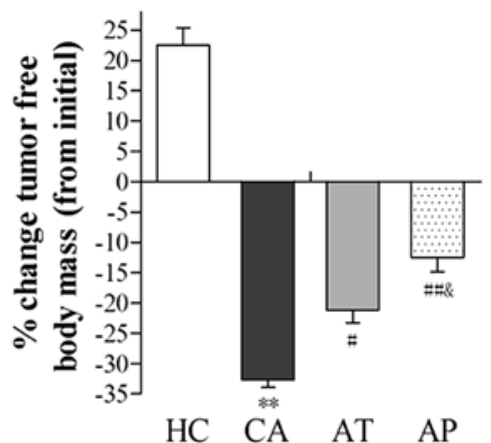

$\mathbf{D}$
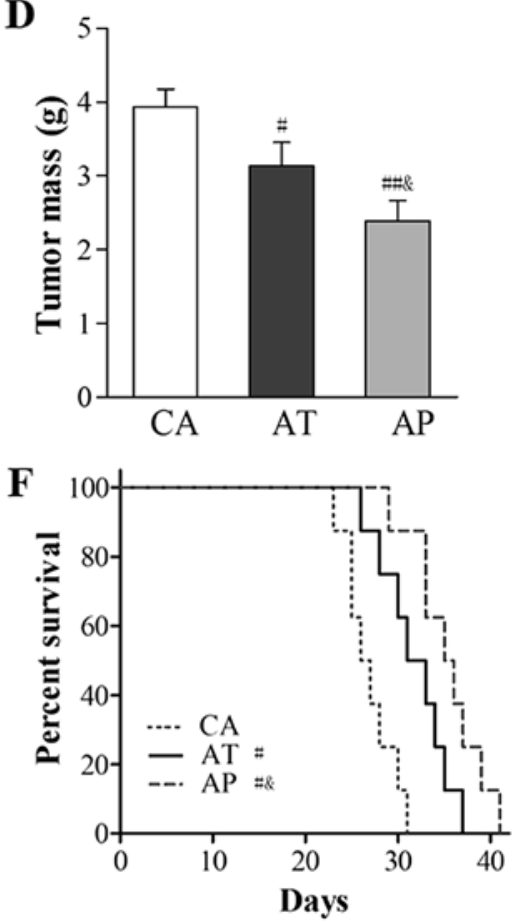

Figure 1. Aliskiren delays cachexia development, reduces tumor burden, and prolongs mouse survival. C26 cells were subcutaneously inoculated into BALB/c mice without any interference ( $n=16$; CA group), those that received early intervention with aliskiren (administered on day 5 after $C 26$ inoculation; $n=16$; AP group), and those that received aliskiren as a therapeutic strategy (administered on day 12 after C26 inoculation; n=16; AT group). As healthy control, PBS was injected subcutaneously into BALB/c mice ( $\mathrm{n}=16$; HC group). (A) The body mass following C26 inoculation was monitored daily and compared among the four groups. (B) On day 20 after C26 inoculation, eight mice from each group were sacrificed and tumors were isolated. The percent change in tumor-free body mass between day 20 and 0 was compared among all four groups. (C) Representative gross images of tumors isolated from mice in the CA, AT, and AP groups are shown. (D) Tumor mass and (E) tumor volume on day 20 were measured and compared among the CA, AT, and AP groups. (F) Survival rates of mice in the CA, AT, and AP groups were analyzed and presented in Kaplan-Meier curves. ${ }^{*} \mathrm{P}<0.05,{ }^{* *} \mathrm{P}<0.01$, compared with the $\mathrm{HC}$ group; ${ }^{\#} \mathrm{P}<0.05$, ${ }^{\# \#} \mathrm{P}<0.01$, compared with the CA group; ${ }^{\&} \mathrm{P}<0.05$, compared with the AT group.

Functionally, aliskiren resulted in significantly improved survival in the AT and AP groups $(\mathrm{P}<0.05$, compared with the CA group), and the best survival was observed in the AP group ( $\mathrm{P}<0.05$, compared to the $\mathrm{CA}$ group; $\mathrm{P}<0.05$, compared to the AT group; Fig. 1F).

Aliskiren improves whole-body strength, mobility and coordination, enhances locomotor activity, and inhibits muscle wasting. Whole-body strength was assessed on day 20 using a grip strength meter and mobility and coordination were assessed using the rotarod test. As shown in Fig. 3A and B, both grip strength and rotarod performance were significantly reduced in the CA group $(\mathrm{P}<0.01$, compared with the HC group). Treatment with aliskiren on day 12 (AT group) improved grip strength and the latency-to-fall during the rotarod test by 21.17 and $16.98 \%$, respectively $(\mathrm{P}<0.05$, compared with the CA group), and prevention using aliskiren on day 5 (AP group) improved these two parameters by 39.06 and $34.56 \%$, respectively $(\mathrm{P}<0.01$, compared with the CA group; $\mathrm{P}<0.05$, compared with the AT group). Similar alterations in locomotor activity also were noticed in different groups; that is, it was dramatically reduced in the CA group $(\mathrm{P}<0.01$, compared with the $\mathrm{HC}$ group), but was enhanced following aliskiren interference $(\mathrm{P}<0.05$, comparing the AT with the CA group; $\mathrm{P}<0.01$, comparing the AP and CA groups), and the best locomotor activity among tumor-bearing mice was observed in the AP group $(\mathrm{P}<0.05$, compared with the AT group; Fig. 2C).

On the histological level, C26 inoculation resulted in marked wasting in skeletal muscles, as represented by reductions in gastrocnemius mass by $40.11 \%$ and in the cross-cut area of gastrocnemius muscle by $36.23 \%$ ( $\mathrm{P}<0.05$, compared with the 

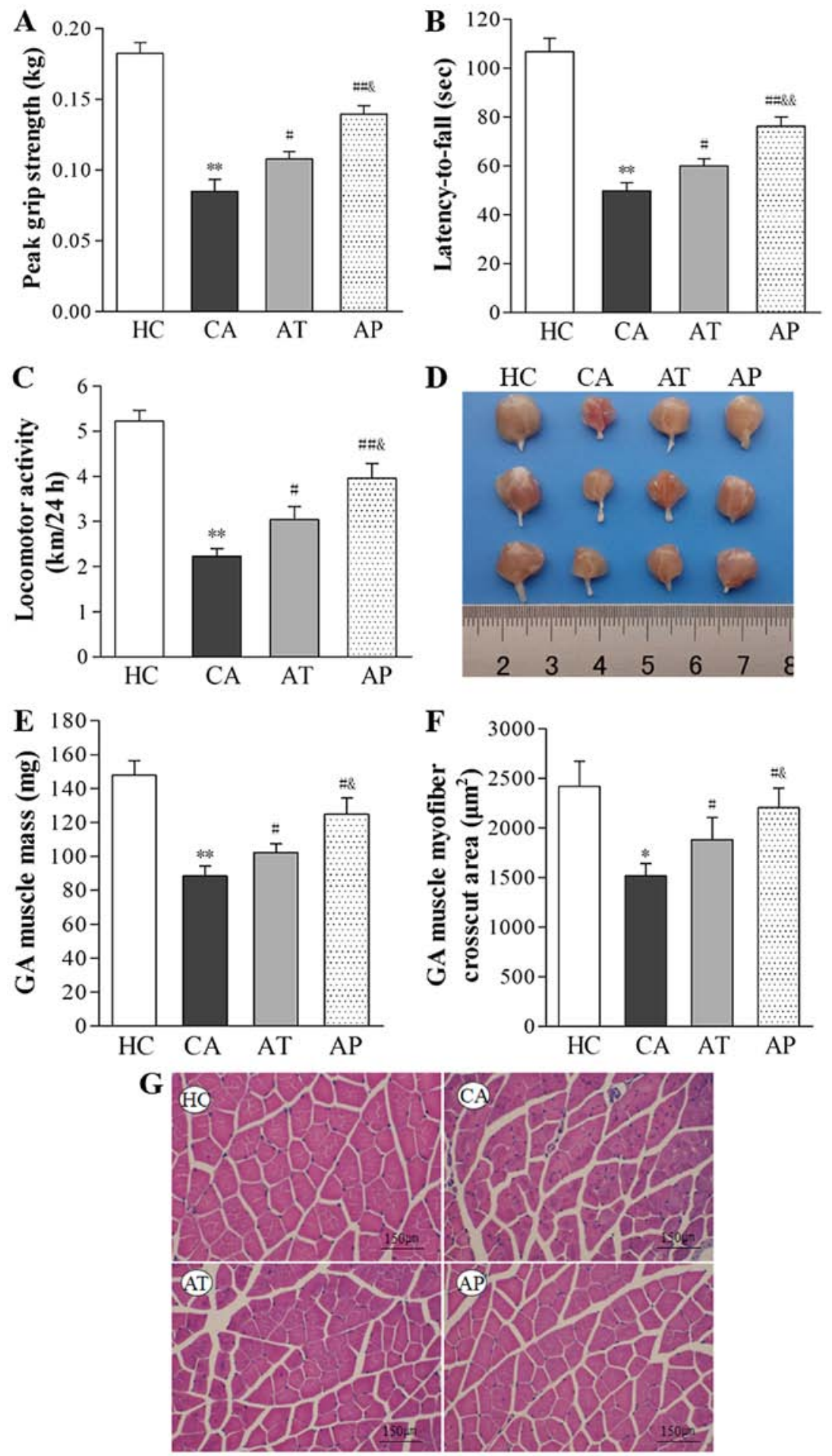

Figure 2. Aliskiren improves whole body strength, mobility, and coordination, enhances locomotor activity, and inhibits muscle wasting. (A) Whole-body grip strength was measured using a grip strength meter, with the maximal value $(\mathrm{kg})$ of five repeats (peak grip strength) for each mouse recorded for data analysis. (B) Whole-body mobility and coordination were assessed using the rotarod performance test, with longest the latency-to-fall (s) value of three repeats for each mouse recorded for data analysis. (C) Locomotor activity was measured over $24 \mathrm{~h}$ for each mouse $(\mathrm{km} / 24 \mathrm{~h})$. (D) Representative gross images of isolated gastrocnemius muscle are shown. (E) The mass of gastrocnemius muscle on day 20 after C26 inoculation was measured and compared between the indicated groups. (F) The cross-cut area of the gasctrocnemius muscle was measured on hematoxylin and eosin (H\&E)-stained sections and compared between the indicated groups. (G) Representative images of H\&E staining (x200 magnification) of gastrocnemius muscle from the indicated groups. "P<0.05, ${ }^{* *} \mathrm{P}<0.01$, compared with the HC group; ${ }^{*} \mathrm{P}<0.05,{ }^{\# \#} \mathrm{P}<0.01$, compared with the $\mathrm{CA}$ group; ${ }^{\text {}} \mathrm{P}<0.05$, \&\& $\mathrm{P}<0.01$, compared with the AT group.

HC group). In contrast, aliskiren increased gastrocnemius mass by $15.47 \%$ in the AT group $(\mathrm{P}<0.05$, compared with the CA group) and $41.14 \%$ in the AP group ( $<<0.05$, compared with the CA group; $\mathrm{P}<0.01$, compared with the AT group), respectively.
It also increased the cross-cut area of gastrocnemius muscle by $24.12 \%$ in the AT group $(\mathrm{P}<0.05$, compared with the CA group) and $41.14 \%$ in the AP group $(\mathrm{P}<0.05$, compared with the CA group; $\mathrm{P}<0.01$, compared with the AT group; Fig. $2 \mathrm{D}-\mathrm{G}$ ). 
Table II. Changes in Ang I and II levels in serum and TNF- $\alpha$ and IL-6 levels in serum and gastrocnemius of mice in the indicated groups $(\mathrm{n}=8$ /group, means $\pm \mathrm{SD})$.

\begin{tabular}{lccccccc}
\hline & & & \multicolumn{2}{c}{ Serum (ng/l) } & & \multicolumn{2}{c}{ Gastrocnemius (pg/mg) } \\
Group & Ang I (ng/l) & Ang II (ng/l) & TNF- $\alpha$ & IL-6 & & TNF- $\alpha$ & IL-6 \\
\hline HC & $9.62 \pm 1.08$ & $33.62 \pm 2.42$ & $14.18 \pm 2.01$ & $2.65 \pm 0.54$ & & $7.24 \pm 1.74$ & $1.27 \pm 0.61$ \\
CA & $25.73 \pm 1.11^{\mathrm{b}}$ & $78.95 \pm 4.15^{\mathrm{b}}$ & $148.42 \pm 10.67^{\mathrm{b}}$ & $91.58 \pm 4.32^{\mathrm{b}}$ & & $52.51 \pm 4.66^{\mathrm{b}}$ & $56.13 \pm 5.57^{\mathrm{b}}$ \\
AT & $14.70 \pm 1.07^{\mathrm{d}}$ & $44.93 \pm 3.31^{\mathrm{d}}$ & $116.00 \pm 16.08^{\mathrm{d}}$ & $64.27 \pm 6.16^{\mathrm{d}}$ & & $22.32 \pm 2.63^{\mathrm{d}}$ & $30.18 \pm 3.01^{\mathrm{d}}$ \\
AP & $13.35 \pm 1.15^{\mathrm{d}}$ & $37.65 \pm 1.83^{\mathrm{d}, \mathrm{e}}$ & $85.85 \pm 10.53^{\mathrm{d}, \mathrm{f}}$ & $45.52 \pm 8.77^{\mathrm{d}, \mathrm{e}}$ & & $15.82 \pm 2.46^{\mathrm{d}, \mathrm{e}}$ & $22.95 \pm 2.38^{\mathrm{d}, \mathrm{e}}$
\end{tabular}

${ }^{\mathrm{a}} \mathrm{P}<0.05,{ }^{\mathrm{b}} \mathrm{P}<0.01$, compared with the $\mathrm{HC}$ group; ${ }^{\mathrm{C}} \mathrm{P}<0.05,{ }^{\mathrm{d}} \mathrm{P}<0.01$, compared with the $\mathrm{CA}$ group; ${ }^{\mathrm{e}} \mathrm{P}<0.05$, ${ }^{\mathrm{f}} \mathrm{P}<0.01$, compared with the AT group. Ang I, angiotensin I; IL-6, interleukin-6; TNF- $\alpha$, tumor necrosis factor- $\alpha$.
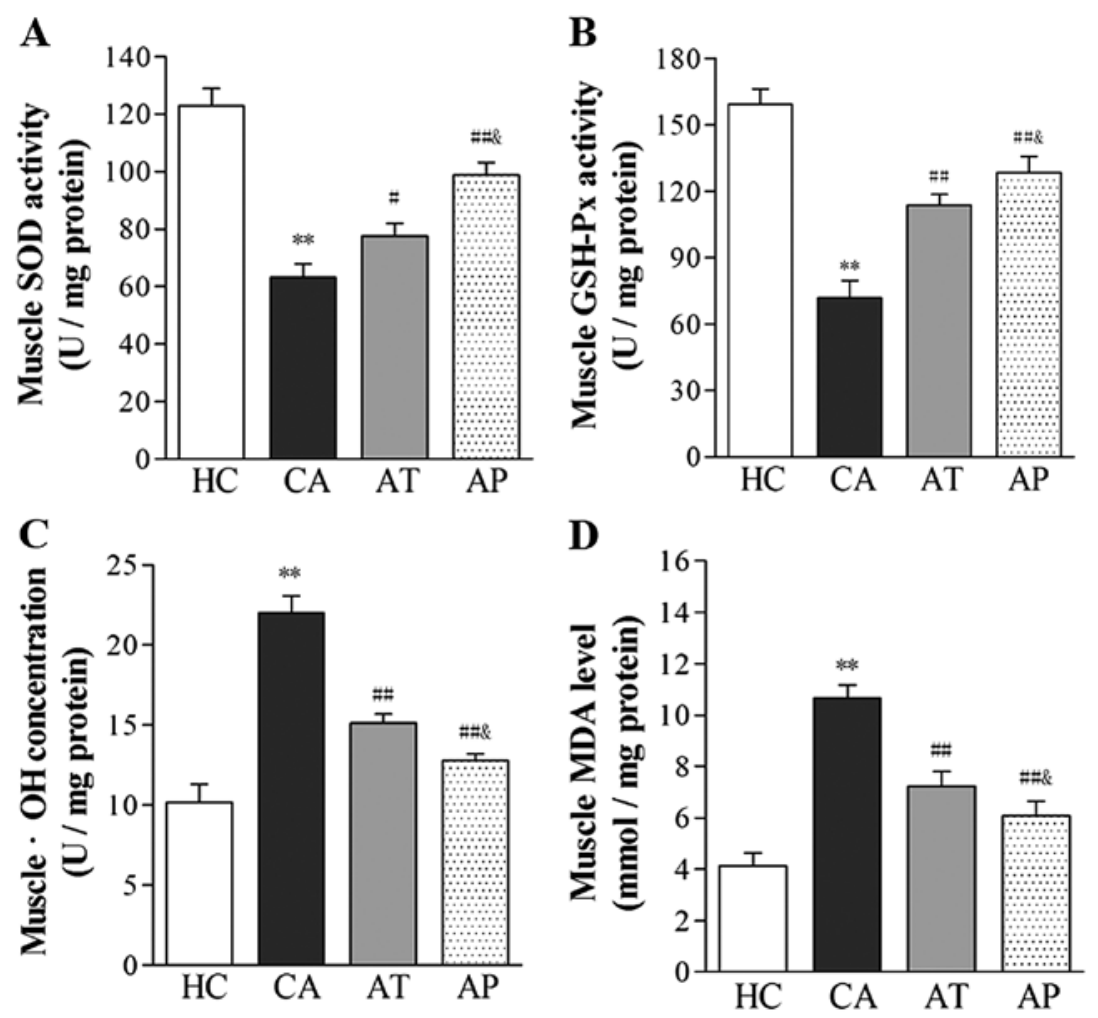

Figure 3. Aliskiren reduces oxidative stress associated with cancer cachexia. The activities of (A) superoxide dismutase (SOD) and (B) glutathione peroxidase (GSH-Px) and the levels of $(\mathrm{C}) \bullet \mathrm{OH}$ and (D) MDA were detected in gastrocnemius muscle from mice of the indicated groups $(\mathrm{n}=8 / \mathrm{group}) .{ }^{* *} \mathrm{P}<0.01$, compared with the HC group; ${ }^{\#} \mathrm{P}<0.05,{ }^{\# \#} \mathrm{P}<0.01$, compared with the CA group; ${ }^{\&} \mathrm{P}<0.05$, compared with the AT group.

Aliskiren inhibits the RAS and controls the upregulation of pro-inflammatory cytokines TNF- $\alpha$ and IL- 6 in both serum and skeletal muscles. To evaluate the effects of aliskiren on the RAS, we measured the serum levels of both Ang I and II, as shown in Table II. The development of cachexia in CA mice was associated with marked RAS activation, as demonstrated by the dramatic elevation of both Ang I and II in serum ( $\mathrm{P}<0.01$, compared with the HC group). In contrast, treatment with aliskiren, either before (AP group) or after (AT group) the development of cachexia, led to serum upregulation of Ang I and II to a much lower degree, with less upregulation observed in the AP group than in the AT group $(\mathrm{P}<0.05)$.

In addition, we also measured the serum and muscular levels of pro-inflammatory cytokines TNF- $\alpha$ as well as IL-6 in all four groups. Similar to the changes in serum Ang I and II levels, TNF- $\alpha$ and IL- 6 levels in both the serum and gastrocnemius muscle were significantly higher in the CA group than in the HC group $(\mathrm{P}<0.01)$. Treatment with aliskiren after cachexia development (AT group) led to reductions in TNF- $\alpha$ and IL-6 in the serum by 21.84 and $29.82 \%$, respectively, and in the muscle by 30.19 and $46.23 \%$, respectively $(\mathrm{P}<0.05$, compared with the CA group). More reductions were detected in the AP group: serum TNF- $\alpha$ and IL-6 by 42.16 and $50.29 \%$, respectively, and muscular TNF- $\alpha$ and IL-6 by 69.87 and $59.11 \%$, respectively ( $\mathrm{P}<0.05$, compared with both the CA and AT groups; Table II).

Aliskiren reduces oxidative stress associated with cancer cachexia. Following the development of cachexia, the level 

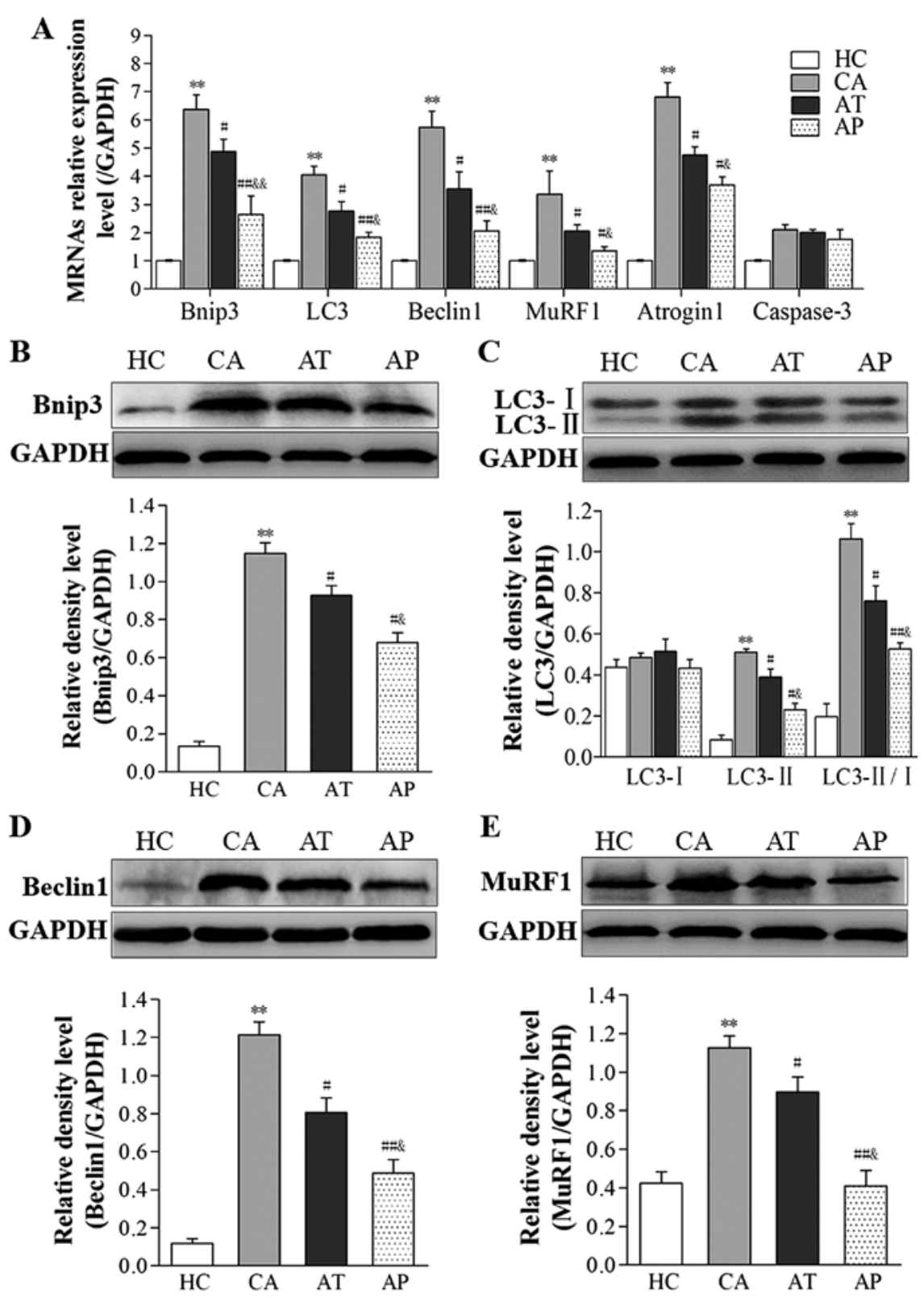

$\mathbf{F}$

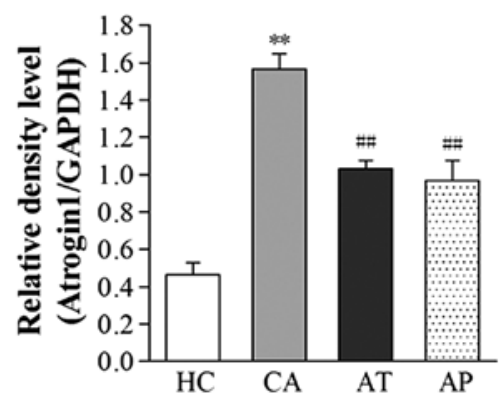

Figure 4. Aliskiren inhibits cachexia-induced autophagy-lysosome pathway (ALP) and ubiquitin-proteasome pathway (UPP) signaling. (A) The steady-state mRNA levels of the indicated genes in gastrocnemius muscle from all four groups were examined by real-time polymerase chain reaction (PCR) and presented as relative ratios to the internal control (GAPDH). (B-F) The protein levels of (B) Bnip3, (C) LC3-I and II, (D) Beclin1, (E) MuRF1, and (F) Atrogin1 were examined by western immunoblotting, and quantified as relative density ratio to that of the internal control (GAPDH). ${ }^{* *} \mathrm{P}<0.01$, compared with the $\mathrm{HC}$ group; ${ }^{\text {" }} \mathrm{P}<0.05,{ }^{\# / 4} \mathrm{P}<0.01$, compared with the CA group; ${ }^{\&} \mathrm{P}<0.05$, \&\& $\mathrm{P}<0.01$, compared with the AT group.

of oxidative stress significantly increased, as indicated by the reduced activity of antioxidative enzymes, SOD and GSH-Px, and the upregulated levels of $\cdot \mathrm{OH}$ and MDA in gastrocnemius muscle ( $\mathrm{P}<0.05$, comparing the $\mathrm{CA}$ and $\mathrm{HC}$ groups). Following aliskiren treatment, the muscular activities of SOD and
GSH-Px increased by 22.91 and $23.92 \%$, respectively, whereas the levels of $\cdot \mathrm{OH}$ and MDA decreased by 31.25 and $32.71 \%$, respectively ( $\mathrm{P}<0.05$, compared with the $\mathrm{CA}$ group). Further reductions in oxidative stress were noted in the AP group, with 56.42 and $37.38 \%$ increases in the muscular activities of SOD 
and GSH-Px, respectively, and 41.86 and $42.93 \%$ decreases in - $\mathrm{OH}$ and MDA levels, respectively $(\mathrm{P}<0.01$, compared with the $\mathrm{CA}$ group; $\mathrm{P}<0.05$, compared with the AT group).

Aliskiren inhibits cachexia-induced ALP and UPP activation. To assess the status of ALP and UPP signaling in cancer cachexia, we examined the expression levels of genes related to these two signaling pathways on both the steady-state mRNA level and protein level. As shown in Fig. 4A, the mRNA levels of autophagy-related genes Bnip3, LC3, and Beclin1, as well as ubiquitin-proteasome-related genes MuRF1 and Atrogin1 in gastrocnemius muscle were significantly upregulated in mice of the CA group compared with these levels in $\mathrm{HC}$ mice $(\mathrm{P}<0.01)$. Upregulation of these genes was dramatically inhibited in the AT and AP groups, with more robust inhibition achieved in the AP group $(\mathrm{P}<0.05$, compared with the AT group). Similar alterations were also observed in the protein levels of these proteins (Fig. 4B-F). In contrast to these changes, the steady-state mRNA level of the apoptosis-related gene caspase- 3 was not markedly altered in tumor-bearing groups (Fig. 4A).

\section{Discussion}

Cachexia is a common complication in patients with malignant tumors and is characterized by increased catabolism, reduced anabolism, and excessive energy expenditure, which leads to progressive multi-organ failure and death. Despite extensive research on cancer cachexia, effective and satisfactory preventive and therapeutic approaches have yet to be developed. Given the significance of the RAS in cancer cachexia, we assessed the effects of a direct renin inhibitor, aliskiren, in this study. Using a mouse model of cancer cachexia, we showed that aliskiren effectively alleviated multiple symptoms associated with cachexia, including weight loss, tumor growth, and muscle wasting. Consequently, aliskiren treatments significantly improved whole-body functions and prolonged mouse survival. Mechanistically, aliskiren antagonizes multiple mechanisms underlying cachexia development, including RAS activation, upregulated systematic inflammation, increased oxidative stress, and enhanced ALP and UPP signaling.

Progressive skeletal muscle wasting is central for cachexia development and is mostly correlated with functional defects in these muscles, which increases the risk of postoperative complications, lowers quality of life, and reduces patient survival (26). Consistently, we showed that following cachexia development in C26 cell-inoculated mice, wasting of skeletal muscles, as demonstrated by loss of gastrocnemius mass and its cross-cut area, was associated with reduced grip strength, whole-body coordination, and locomotor activity. The administration of aliskiren as a therapeutic agent and more robustly as a preventive agent, significantly ameliorated muscle wasting and skeletal muscle functions, implying its prophylactic and therapeutic potential for cancer cachexia.

Chronic inflammation is an important feature of cancer cachexia. The inflammatory cytokines TNF- $\alpha$ and IL- 6 play critical pathogenic roles in the development of cancer cachexia via multiple mechanisms, inducing anorexia, promoting muscular protein degradation, inhibiting protein synthesis, and enhancing energy expenditure (27-29). Recent studies demonstrated that aliskiren achieves its anti-inflammatory property by inhibiting the RAS and reducing the release of inflammatory mediators $(18,20)$. Consistent with these results, we showed that administration of aliskiren as a preventive or therapeutic agent significantly reduced the levels of TNF- $\alpha$ and IL- 6 in both serum and skeletal muscle, suggesting anti-inflammation as a second mechanism for the anti-cachexia activity of aliskiren.

Oxidative stress is another important mechanism contributing to the development of anorexia and loss of skeletal muscles in cancer patients. Several causes have been linked to the pathogenesis of oxidative stress in cancer cachexia, including: i) anorexia reduces the intake of antioxidants; ii) alterations in metabolism reduces the synthesis of reducing agents; iii) upregulation of pro-inflammatory cytokines, such as TNF- $\alpha$ and IL-6, increases the production of reactive oxygen species (ROS); and iv) some anticancer medicines such as alkylating agents boost ROS generation (30). Of the endogenous antioxidants, SOD and GSH-Px are two important enzymes. Their activities reflect the body's capability to clear free radicals and protect against oxidative damage. MDA results from lipid degradation by ROS, and together with $\cdot \mathrm{OH}$, indicates the level of oxidative stress in an organism. In this study, we showed that cachexia led to significant downregulation of SOD and GSH-Px but upregulation of MDA and $\cdot \mathrm{OH}$ in skeletal muscles, indicating an increase in oxidative stress. Aliskiren administration significantly reduced the level of oxidative stress in the mice by enhancing the activities of SOD and GSH-Px while reducing the levels of MDA and $\cdot \mathrm{OH}$. Consistently, Patel et al (19) and Thomas et al (31) demonstrated that aliskiren can inhibit oxidative stress by reducing the generation of free radicals. Therefore, targeting oxidative stress provides a third mechanism underlying the anti-cachexia activity of aliskiren.

Protein degradation in skeletal muscles under cancer cachexia is achieved mainly through two signaling pathways, ALP and UPP $(32,33)$. Chronic inflammation and oxidative stress associated with cancer cachexia could upregulate autophagy-related genes and activate $\operatorname{ALP}(34,35)$. In this study, we found that cachexia development in mice led to significant upregulation of the ALP-related genes Bnip3, LC3 (increased LC3-II/LC3-I ratio), and Beclin1, indicating ALP activation. Following aliskiren administration, expression of these genes was markedly downregulated, implying reduced ALP activity. This finding is consistent with those of Weng et al and Zhang et al who reported that aliskiren inhibits ALP activity $(36,37)$. Atrogin-1 and MuRF1 are two muscle-specific E3 ligases that critically control UPP activity, and the inhibition of these two enzymes has been demonstrated to alleviate the loss of skeletal muscles and ameliorate cancer cachexia $(38,39)$. Here we showed that aliskiren reduced the mRNA expression levels of these two enzymes and thus improved cancer cachexia.

In summary, aliskiren is highly effective at ameliorating multiple symptoms associated with cancer cachexia. It inhibits weight loss, wasting of skeletal muscles, and whole-body dysfunctions, and results in improved locomotor activities as well as prolonged survival. On the molecular level, aliksiren targets multiple mechanisms underlying the pathogenesis of cancer cachexia, including RAS activation, 
chronic inflammation, increased oxidative stress, and stimulation of ALP and UPP signaling. Early administration of aliskiren as a preventive agent (before the development of cachexia) offers more robust anti-cachexia activities than later administration as a therapeutic agent (after the development of cachexia). This study proves the efficacy of aliskiren as an anti-cachexia agent in an experimental animal model, and the results support further testing of this agent in cancer patients in clinical settings.

\section{References}

1. Fearon K, StrasserF, AnkerSD,Bosaeus I,Bruera E, FainsingerRL, Jatoi A, Loprinzi C, MacDonald N, Mantovani G, et al: Definition and classification of cancer cachexia: An international consensus. Lancet Oncol 12: 489-495, 2011.

2. Tan BH and Fearon KC: Cachexia: Prevalence and impact in medicine. Curr Opin Clin Nutr Metab Care 11: 400-407, 2008.

3. Tisdale MJ: Mechanisms of cancer cachexia. Physiol Rev 89: 381-410, 2009.

4. Russell ST, Sanders PM and Tisdale MJ: Angiotensin II directly inhibits protein synthesis in murine myotubes. Cancer Lett 231: 290-294, 2006

5. Sanders PM, Russell ST and Tisdale MJ: Angiotensin II directly induces muscle protein catabolism through the ubiquitin-proteasome proteolytic pathway and may play a role in cancer cachexia. Br J Cancer 93: 425-434, 2005

6. Ruiz-Ortega M, Lorenzo O, Suzuki Y, Rupérez M and Egido J: Proinflammatory actions of angiotensins. Curr Opin Nephrol Hypertens 10: 321-329, 2001.

7. Zhang L, Du J, Hu Z, Han G, Delafontaine P, Garcia G and Mitch WE: IL-6 and serum amyloid A synergy mediates angiotensin II-induced muscle wasting. J Am Soc Nephrol 20: 604-612, 2009.

8. Russell ST, Eley $\mathrm{H}$ and Tisdale MJ: Role of reactive oxygen species in protein degradation in murine myotubes induced by proteolysis-inducing factor and angiotensin II. Cell Signal 19: 1797-1806, 2007

9. Sukhanov S, Semprun-Prieto L, Yoshida T, Michael Tabony A, Higashi Y, Galvez S and Delafontaine P: Angiotensin II oxidative stress and skeletal muscle wasting. Am J Med Sci 342: 143-147, 2011.

10. Dalla Libera L, Ravara B, Angelini A, Rossini K, Sandri M, Thiene G, Battista Ambrosio G and Vescovo G: Beneficial effects on skeletal muscle of the angiotensin II type 1 receptor blocker irbesartan in experimental heart failure. Circulation 103 . 2195-2200, 2001.

11. Neo JH, Ager EI, Angus PW, Zhu J, Herath CB and Christophi C: Changes in the renin angiotensin system during the development of colorectal cancer liver metastases. BMC Cancer 10: 134, 2010.

12. Sun G, Haginoya K, Dai H, Chiba Y, Uematsu M, Hino-Fukuyo N, Onuma A, Iinuma $\mathrm{K}$ and Tsuchiya $\mathrm{S}$ : Intramuscular renin-angiotensin system is activated in human muscular dystrophy. J Neurol Sci 280: 40-48, 2009

13. Wang XH and Mitch WE: Mechanisms of muscle wasting in chronic kidney disease. Nat Rev Nephrol 10: 504-516, 2014.

14. Murphy KT, Chee A, Trieu J, Naim T and Lynch GS: Inhibition of the renin-angiotensin system improves physiological outcomes in mice with mild or severe cancer cachexia. Int J Cancer 133 1234-1246, 2013.

15. Wang Q, Li C, Peng X, Kang Q, Deng D, Zhang L, Zheng Y, Wang C, Qiao Z, Guo D, et al: Combined treatment of carfilzomib and $\mathrm{z}$-VAD-fmk inhibits skeletal proteolysis and apoptosis and ameliorates cancer cachexia. Med Oncol 32: 100, 2015.

16. Acharyya S and Guttridge DC: Cancer cachexia signaling pathways continue to emerge yet much still points to the proteasome. Clin Cancer Res 13: 1356-1361, 2007.

17. Penna F, Baccino FM and Costelli P: Coming back: Autophagy in cachexia. Curr Opin Clin Nutr Metab Care 17: 241-246, 2014.

18. Del Fiorentino A, Cianchetti S, Celi A and Pedrinelli R: Aliskiren, a renin inhibitor, downregulates TNF- $\alpha$-induced tissue factor expression in HUVECS. J Renin Angiotensin Aldosterone Syst 11: 243-247, 2010.
19. Patel RB, Prajapati KD, Sonara BM, Sharma MM, Patel HM, Pawar VD and Jain MR: Ameliorative potential of aliskiren in experimental colitis in mice. Eur J Pharmacol 737: 70-76, 2014.

20. Schmerbach K, Pfab T, Zhao Y, Culman J, Mueller S, Villringer A, Muller DN, Hocher B, Unger T and Thoene-Reineke C: Effects of aliskiren on stroke in rats expressing human renin and angiotensinogen genes. PLoS One 5: e15052, 2010.

21. De Luca A, Pierno S, Liantonio A, Cetrone M, Camerino C, Fraysse B, Mirabella M, Servidei S, Rüegg UT and Conte Camerino D: Enhanced dystrophic progression in $\mathrm{mdx}$ mice by exercise and beneficial effects of taurine and insulin-like growth factor-1. J Pharmacol Exp Ther 304: 453-463, 2003.

22. Ham DJ, Murphy KT, Chee A, Lynch GS and Koopman R: Glycine administration attenuates skeletal muscle wasting in a mouse model of cancer cachexia. Clin Nutr 33: 448-458, 2014.

23. Murphy KT, Chee A, Trieu J, Naim T and Lynch GS: Importance of functional and metabolic impairments in the characterization of the C-26 murine model of cancer cachexia. Dis Model Mech 5: $533-545,2012$.

24. Schmittgen TD and Livak KJ: Analyzing real-time PCR data by the comparative C(T) method. Nat Protoc 3: 1101-1108, 2008

25. Tanaka Y, Eda H, Tanaka T, Udagawa T, Ishikawa T, Horii I, Ishitsuka H, Kataoka T and Taguchi T: Experimental cancer cachexia induced by transplantable colon 26 adenocarcinoma in mice. Cancer Res 50: 2290-2295, 1990.

26. Guo CB, Zhang W, Ma DQ, Zhang KH and Huang JQ: Hand grip strength: An indicator of nutritional state and the mix of postoperative complications in patients with oral and maxillofacial cancers. Br J Oral Maxillofac Surg 34: 325-327, 1996.

27. Carson JA and Baltgalvis KA: Interleukin 6 as a key regulator of muscle mass during cachexia. Exerc Sport Sci Rev 38: 168-176, 2010.

28. Deans C and Wigmore SJ: Systemic inflammation, cachexia and prognosis in patients with cancer. Curr Opin Clin Nutr Metab Care 8: 265-269, 2005

29. Tsoli M and Robertson G: Cancer cachexia: Malignant inflammation, tumorkines, and metabolic mayhem. Trends Endocrinol Metab 24: 174-183, 2013.

30. Vaughan VC, Martin P and Lewandowski PA: Cancer cachexia: Impact, mechanisms and emerging treatments. J Cachexia Sarcopenia Muscle 4: 95-109, 2013.

31. Thomas CM, Yong QC, Seqqat R, Chandel N, Feldman DL, Baker KM and Kumar R: Direct renin inhibition prevents cardiac dysfunction in a diabetic mouse model: Comparison with an angiotensin receptor antagonist and angiotensin-converting enzyme inhibitor. Clin Sci (Lond) 124: 529-541, 2013.

32. Penna F, Costamagna D, Pin F, Camperi A, Fanzani A, Chiarpotto EM, Cavallini G, Bonelli G, Baccino FM and Costelli P: Autophagic degradation contributes to muscle wasting in cancer cachexia. Am J Pathol 182: 1367-1378, 2013.

33. Tardif N, Klaude M, Lundell L, Thorell A and Rooyackers O: Autophagic-lysosomal pathway is the main proteolytic system modified in the skeletal muscle of esophageal cancer patients. Am J Clin Nutr 98: 1485-1492, 2013.

34. McClung JM, Judge AR, Powers SK and Yan Z: p38 MAPK links oxidative stress to autophagy-related gene expression in cachectic muscle wasting. Am J Physiol Cell Physiol 298: C542-C549, 2010.

35. Rahman M, Mofarrahi M, Kristof AS, Nkengfac B, Harel S and Hussain SN: Reactive oxygen species regulation of autophagy in skeletal muscles. Antioxid Redox Signal 20: 443-459, 2014.

36. Weng LQ, Zhang WB, Ye Y, Yin PP, Yuan J, Wang XX, Kang L, Jiang SS, You JY, Wu J, et al: Aliskiren ameliorates pressure overload-induced heart hypertrophy and fibrosis in mice. Acta Pharmacol Sin 35: 1005-1014, 2014.

37. Zhang W, Zhao G, Hu X, Wang M, Li H, Ye Y, Du Q, Yao J, Bao Z, Hong W, et al: Aliskiren-attenuated myocardium apoptosis via regulation of autophagy and connexin-43 in aged spontaneously hypertensive rats. J Cell Mol Med 18: 1247-1256, 2014.

38. Burckart K, Beca S, Urban RJ and Sheffield-Moore M: Pathogenesis of muscle wasting in cancer cachexia: Targeted anabolic and anticatabolic therapies. Curr Opin Clin Nutr Metab Care 13: 410-416, 2010

39. Yuan L, Han J, Meng Q, Xi Q, Zhuang Q, Jiang Y, Han Y, Zhang B, Fang J and Wu G: Muscle-specific E3 ubiquitin ligases are involved in muscle atrophy of cancer cachexia: An in vitro and in vivo study. Oncol Rep 33: 2261-2268, 2015. 